\title{
Co-writing Values: What We Did and Why We Did It
}

\author{
Bill Fulford
}

\subsection{Introduction}

This book is ambitious. It was ambitious in its original scope and aims. It has been ambitious in implementation. It is ambitious for the future.

In this concluding chapter, we describe the processes we adopted in pursuing our ambitions for the book and take stock of its outcomes. We look first, in Sect. 2, at the challenges that were presented by the co-writing approach that we adopted. Section 3 then describes how we deployed the resources of values-based practice in addressing these challenges. Finally, Sect. 4 reflects on outcomes. We offer no further justification for our ambitious co-writing approach than the range and quality of the contributions to the book. We conclude with a brief reflection on the implications of the book for the future of person-centred care not just in mental health but in health care as a whole.

\subsection{What We Did (a)-Finding the Challenges}

As we described in our Preface, this book came about as an unintended though welcome consequence of the 'embarrassment of riches' of personal narratives that resulted from our initial call for contributions. Faced with the wide diversity of these contributions, it became immediately clear that as Editors (being, although geographically diverse, all white, male and psychiatrists), we were ill-equipped to represent the book as a whole.

\footnotetext{
Authors

The Editors with input from all contributors

B. Fulford $(\bowtie)$

St Catherine's College, University of Oxford, Oxford, UK

(C) The Author(s) 2021

D. Stoyanov et al. (eds.), International Perspectives in Values-Based Mental

Health Practice, https://doi.org/10.1007/978-3-030-47852-0_47
} 
Various options for remedying this were considered at an initial writing workshop convened by the Lead Editor, Drozdstoj Stoyanov, and hosted by one of our contributors, Hasanen Al-Taiar, under the auspices of the Royal College of Psychiatrists in London. It was this workshop that settled on co-writing as a valuesbased strategy for capturing the full range and diversity of the proposed contributions to the book. A second workshop then took this strategy forward by developing a shared framework of values within which as co-writing contributors we would all work. (The second workshop is described further in Sect. 3.)

\subsubsection{First Workshop: Raising Awareness of Values}

The first workshop brought together a small group recruited, for practical reasons, locally from among those able to attend but broadly reflecting the range of backgrounds and experiences of contributors to the book as a whole.

The programme for the day started with two exercises routinely used in valuesbased practice training sessions to raise awareness of values and of differences of values. ${ }^{1}$ The aim at the time was to give our contributors an introduction to values-based practice by experiencing it for themselves. But as is so often the way with exercises in values-based practice we got more than we had bargained for! So effective were the exercises in raising awareness of values and (more particularly) of differences of values that a number of (sometimes painful) clashes of values emerged among participants.

The depth of the challenges presented by these clashes of values is illustrated by the sample comments that follow. These reflect the range of comments on one of the dominant themes, Respect and Disagreement. They are partly as made in the first workshop, partly as reprised in the second, and partly as subsequently submitted by way of follow-up. ${ }^{2}$ We return to some of the other themes below (see Risks, Resources and Strengths). Although anonymized, the comments are reproduced with the permission of those concerned.

- Speaker 1 - is the resolution that XX (whose values had upset and offended some participants) resigns?

- Speaker 2-my reaction is the opposite-I agree this has made for an uncomfortable meeting but it is good that things have been said openly so they can be dealt with rather than get sat on.

- Speaker 3-if the book as a whole doesn't represent my values I will withdraw but this doesn't mean I agree with everything in every chapter-so its about facing our challenges-not an easy thing to do-but we have to agree to acknowledge differences.

\footnotetext{
${ }^{1}$ The exercises used were the 'three words' exercise and the 'forced choice' exercise- these are described fully in the sources indicated in the Guide to Further Information at the end of this chapter.

${ }^{2}$ The extracts given here are representative of the comments and views expressed on the theme of Respect and Disagreement in the course of a much longer discussion (continuing in all for over an hour and a half across the two workshops). The numbering of the speakers is solely for purposes of differentiating them and does not reflect the order in which they spoke.
} 
- Speaker 2-my standards are 'am I proud of my contribution and will my contribution make a difference?'

- Speaker 3-there is an important difference between disagreeing and taking offense. We must behave in a way that is consistent with the book so may agree to disagree but so long as we all believe in values-based practice, and in actually doing it, a key part of what we are about is to share and acknowledge differences.

- Speaker 2-my desire to say what I want to say may sit alongside things I think are wrong.

- Speaker 3-it's not about agreement but about finding that framework of respect within which we may disagree without taking offense.

Through this and similar discussions, we found the originally planned 'introduction to values-based practice' morphed into a substantive and as it proved particularly intense exercise in values-based practice itself. We took this exercise forward, following the last speaker's suggestion, with the development in the second workshop of a shared framework of values.

\subsection{What We Did (b): Facing the Challenges}

The second workshop-kindly convened by another of our contributors, David Crepaz-Keay, at the Mental Health Foundation ${ }^{3}$ - focused as we have said on establishing a framework of shared values within which we could all agree to work in taking the book forward.

As a key component of values-based practice, such frameworks should be established on a locally agreed basis among those directly concerned (see Table 1.2, chapter 1, "Surprised by Values: An Introduction to Values-Based Practice and the Use of Personal Narratives in this Book"). It was just such a locally agreed framework that the second workshop gave us the opportunity to produce. Led on this occasion by Kim Woodbridge-Dodd and Evette Hunkins-Hutchinson (authors of the reflective chapter 44, "Reflections on the Impact of Mental Health Ward Staff Training in Race Equality and Values-Based Practice"), the group agreed to adopt a modified version of a framework of shared values developed some years ago to support the policy implementation work of NIMHE [1]. ${ }^{4}$ Those present contributed suggestions for modifying this framework. It was then circulated for comment to the group as a whole. The version finally adopted is given in Fig. 47.1.

\footnotetext{
${ }^{3}$ The Mental Health Foundation is a mental health NGO based in London (see https://www. mentalhealth.org.uk). David, who is the author of chapter 22 "Three Points in Time: How Values and Culture Affected my Life, Madness and the People Around Me" and first author of chapter 28 "Journey into Genes: Cultural Values and the (Near) Future of Genetic Counselling in Mental Health", is the Head of Empowerment and Social Inclusion at MHF.

${ }^{4}$ NIMHE (the National Institutes for Mental Health, England) was a policy implementation body set up by the UK's Department of Health some years ago to support what at the time was an ambitious programme of mental health services reform. NIMHE's first Director, Anthony Sheehan, convened an interdisciplinary group (of which one of us, KWMF, was a member) tasked with producing a Framework of Values to guide its work.
} 


\section{The modified NIMHE Values Framework adopted in developing the book}

The work of the editors and authors will be guided by three principles of valuesbased practice:

1) Recognition - as editors and authors we will recognize the role of values in all areas of mental health policy and practice and will aim to reflect this in developing the book.

We recognize that no judgement can be 'value free' but results from such factors as class, background and culture.

2) Raising Awareness - as editors and authors we will be committed to raising awareness of the values involved in different contexts, the role/s they play and their impact on the development of this book.

Our commitment to raising awareness of values will include recognizing our own values and how these reflect our very different backgrounds and life experiences. It will also include acknowledging the privilege we have of being able to adopt and write from our respective first-personal perspectives.

3) Respect - as editors and authors we will respect the diversity of values and will be committed to working with such diversity that makes the principle of person centrality a unifying focus for practice.

This principle means that the values of each individual and their communities must be the starting point and key determinant for all understandings and decisions by the editors and authors involved in the book.

The Principle of Respect has a number of other important implications for the way we develop this book

- A commitment to dialogue - we believe that the book must evidence our commitment to dialogue between professionals, service users and carers that recognizes the unique contributions each brings.

- Equality of citizenship - respect for diversity of values encompasses a number of specific policies and principles concerned with equality of citizenship and it is acknowledged it will be enacted with different levels and forms of interpersonal and collective power relations.

- Equality between peoples - We recognize that equality of citizenship may be constituted differently within different nations and cultures, and that. as this is an international publication, this will in itself introduce a range of ways of understanding the meanings and expectations of what equality is and of how respectful behavior is demonstrated.

- No go areas - We share the view that respect for diversity requires antidiscriminatory attitudes and behaviors because discrimination in all its forms is intolerant of diversity. Thus respect for diversity of values has the consequence that it is unacceptable to discriminate on grounds such as gender, sexual orientation, class, age, abilities, religion, race, culture or language.

- Our overall approach - In aiming to embody respect for diversity in developing this book we will:

Fig. 47.1 The modified NIMHE Values Framework adopted in developing the book 
- Be prepared to listen to and make the required effort to gain insight into how other people different from ourselves understand a given situation

o Reflectively combine self-monitoring and self-management with positive self-regard

- Believe it is possible to be respectful of our own individual humanity and vulnerability while at the same time, 1) recognizing that others may see things differently, and 2) accepting that others may hold views with which we disagree but nonetheless respect.

- Recognize that working with values is relational and may evoke strong personal emotions.

- We will give time and space to: 1) becoming aware of the different values at play; 2) being open, respectful and responsive to others values; and 3 ) balancing, where possible, the differing values to the best mutually constructive outcome.

Fig. 47.1 (continued)

\subsubsection{Risks, Resources and Strengths}

Although agreeing a Framework of Shared Values was a good start, there was considerably more to the process of co-writing the book. This required 'above and beyond' engagement from our contributors and an additional administrative load for Springer. There were multiple exchanges of drafts of individual chapters and of large sections of the book as a whole. Where differences arose, there were balancing compromises to find. These were important particularly in the light of the issues of Power and of Language Use/Communication that were extensively aired in both workshops. For example:

- Speaker 6-equality between authors and editors means genuine two-way communication between people on an equal footing.

- Speaker 7-Is the book for psychiatrists or the general public? If the latter we must use language that anyone can understand.

- Speaker 6-we are the 'we' here-this is about what can 'we' do together-we are all stakeholders in the process.

- Speaker 2-I spend much of my time on projects where I feel the professionals present have no real idea what it means to be a psychiatric patient.

- Speaker 4-the point is to acknowledge and deal with the issues of power that come up-this is something that we can do something about-it is, literally, within our power.

- Speaker 1-yes, the power to work through the issues - that at least should be the intention of the editing process.

- Speaker 5-the issues of editorial power run both ways-the editors have to manage issues of academic power (such as peer review and citation ratings) as well as managing power relationships with authors.

- Speaker 1-the challenge is to have an impact - this requires successfully getting the book out and published but without compromising what we are trying to say. 
As editors, we thus had to rethink our role in the book, stepping back and relinquishing a part of the control we would normally have exercised. There were risks in this of course. We could have lost contributors (we did lose one). But such risks were more than compensated for by the many ways in which contributors supported the task of implementation. It was very helpful for a start that they were realistic about just how difficult this was likely to be:

- Speaker 3-We know from experience that values-based practice isn't easy to implement.

- Speaker 1-a 2009 report from the Coalition Government puts service users first-but again it simply isn't happening—so if it doesn't happen with this book is it a con?

- Speaker 3-even here [in co-writing the book] we started with respect but when two people had different points of view everyone felt threatened-if we can't get it right in this room how can we expect to get service users'values acted on in the wider community.

Notwithstanding the difficulties, however, contributors engaged positively with the process. Indeed, the dissensus ${ }^{5}$ from which the book gradually emerged was managed as much by our co-writing contributors as by us as editors. In the first writing workshop, for example when emotionally distressing issues were raised, it was one of our contributors who brought the group together around our shared commitment to the mutual respect underpinning values-based practice. Contributors also came up with many practical ideas and assets:

- Speaker 7-one way to have an impact is to build on the [VBP] Centre's and other resources established by different cultural groups-instead of giving us services, give us the money and control to set up our own services.

- Speaker 8-the learning from the book has to be structured to link up with what works-there are excellent cultural competency trainers (for example at the Steven Laurence Centre in London) and helpful publications (such as Robin Diangelo's "White Fragility", [2]) that really ring bells about the language that's used in psychiatry.

- Speaker 9-the engagement itself has to be sensitive to cultural values-for example there was a theatre project 20 years ago in London that involved a young director from the Brazilian favelas [slums] being asked to put on one of his productions - like most Brazilians his way of working included physical contact so he was devastated when in a break one of the theatre officials told him 'we invited you here to do your theatre but here we don't touch people'.

Many further resources for implementation are included in the sources given in the Guides to Further Information at the ends of individual chapters.

\footnotetext{
${ }^{5}$ See Table 1.1 in the Introduction to Values-based Practice in chapter 1, "Surprised by Values: An Introduction to Values-Based Practice and the Use of Personal Narratives in this Book".
} 


\subsection{Why We Did It}

Exceptional claims, as the familiar aphorism has it, require exceptional proofs. Our central claim in this book (that cultural values are a key factor in the causes, presentation and management of mental health issues), and the means by which we have chosen to establish that claim (by way of personal narratives), may seem to be, in the sense of the aphorism, 'exceptional'. At the very least it may be said, some justification is required for the extent to which the approach adopted diverges from the 'tried and tested' norms of contemporary scientific medicine.

\subsubsection{Justifying Values?}

We have offered the beginnings of the required justification in our opening chapter with our proposed inversion of the evidence hierarchy for values: personal narratives we argued may come at the bottom of the evidence hierarchy but they should come at the very top of the corresponding hierarchy for values (chapter 1, "Surprised by Values: An Introduction to Values-Based Practice and the Use of Personal Narratives in this Book", Fig. 1.3). This inversion we further argued, far from alienating values-based practice from evidence-based practice is an aspect of and directly reflects the partnership between them in clinical decision-making.

A full working out of this justification is beyond our scope here. Such working out might start with the history of ideas, looking at the (traditionally recognized) sources of contemporary understandings of mental health issues in the outputs from the Nineteenth Century Methodonstreit, and the influences of these sources on Karl Jaspers in developing his distinction between causal explanations and meaningful understanding of psychopathology. In the light of the contributions to this book, moreover, sources from the history of ideas should be extended beyond those traditionally cited to include, for example, work on the effects of colonization and slavery on norms of rationality, and, hence, how psychiatric diagnostic concepts are used in practice (see, for example, among other pertinent chapters in this book, chapters 11, "Madness, Mythopoetry and Medicine", 18, "Colonial Values and Asylum Care in Brazil: Reclaiming the Streets Through Carnival in Rio de Janeiro", 20, "Living at the Edge of Compromise: Balkan Pluralism as a Resource for Balanced Decision-Making" and Colin King's contribution to chapter 46, "Beyond the Color Bar: Sharing Narratives in Order to Promote a Clearer Understanding of Mental Health Issues Across Cultural and Racial Boundaries").

These sources might in turn lead to their counterparts in various areas of contemporary philosophy: to the philosophy of mind, for example, with work on levels of explanation and its applications across such areas as information theory, artificial intelligence and the neurosciences; to moral theory, notably in on-going debates about the logical relationship (the relationship of meaning) between assertions of fact and of value; and to cross-disciplinary work between the philosophy and sociology of science. Sources for all these and other relevant areas are given in the Guide to Further Information at the end of the chapter. 


\subsubsection{A Provisional Justification}

The theoretical issues involved in grounding values-based practice on personal narratives are thus all too real. This is why as we noted in the introduction to Part II (chapter 6, "Theory First: An Introduction to Part II, Theory"), theory plays such a large role in values-based practice and in this book. For present purposes however, we believe our approach is at least provisionally justified by the diverse range and quality of the contributions to the book.

We return below to why we say 'provisionally justified' but a quick caste across the contents of the book is sufficient to show that its contributions are indeed nothing if not diverse. There is wide diversity in style and content. There is wide diversity of geographical regions and of cultural and ethnic representation. There is wide diversity of topics covering all the major forms of mental health issues and a range of treatment approaches (including self-management). There is, importantly, wide diversity of expertise including (with equal authority of voice) expertise by experience and expertise by training. Such diversity might suggest an inchoate storyline. But the contributions as we described in our introductory chapter 1 (Sect. 4), fell naturally into the established framework structure of values-based practice.

\subsubsection{Why Diversity of Perspectives Is Important Clinically}

The diversity of perspectives represented by the contributions to this book is important clinically. It is important across the board in the shared clinical decision-making that is the basis of contemporary person-centred clinical care. This is why valuesbased practice, offering as it does a way of engaging effectively with diverse value perspectives, is important across the board in health care (remember the story from orthopaedic surgery in chapter 1, "Surprised by Values: An Introduction to ValuesBased Practice and the Use of Personal Narratives in this Book" about Mrs. Jones and her arthritic knee).

In mental health, this same diversity of perspectives has the further importance of providing a foil against abuse. Mental health interventions are notoriously vulnerable to being used abusively for purposes of political or personal control [3]. There are reasons (contested reasons to be sure) for believing that the vulnerability of mental health to abuse of this kind arises from the influence of unacknowledged values on judgements of rationality [4]. The influence of such values is evident in several chapters in this book: for example, in chapter 18, "Colonial Values and Asylum Care in Brazil: Reclaiming the Streets Through Carnival in Rio de Janeiro" with the impact on unacknowledged colonial values on attitudes to mental health in Brazil, chapter 27, "Nontraditional Religion, Hyper-religiosity, and Psychopathology: The Story of Ivan from Bulgaria" with the impact of unacknowledged values of rationality on the interpretation of DSM's 'criteria of clinical significance', and chapter 46, "Beyond the Colour Bar: Sharing Narratives in Order to 
Promote a Clearer Understanding of Mental Health Issues Across Cultural and Racial Boundaries" with the impact of unacknowledged values of whiteness on racially disproportionate uses of involuntary psychiatric treatment in the UK. Other factors may be involved in abusive uses of psychiatry becoming institutionalized [5]. But it is in unacknowledged cultural values, so this line of reasoning goes, that the essential vulnerability of mental health to abuse has its origins.

In raising awareness of values, values-based practice thus has a role to play in mental health not only in promoting good practice (by supporting shared clinical decision making) but also in preventing bad practice (as a foil against abuse). But raising awareness of one's own values is difficult: recall our own surprise in chapter 1 , at the extent to which our unacknowledged values of individualism had influenced the original development of values-based practice. Which is where the diversity of perspectives represented by the contributions to this book comes crucially into play. For just as in chapter 1, exposure to a diversity of value perspectives prompted insight into the way our own unacknowledged values had shaped valuesbased practice, so, too, may exposure to a diversity of value perspectives prompt similar insights into the ways unacknowledged values continue to shape approaches to mental health issues.

\subsubsection{Why Diversity of Perspectives Has Been Important in This Book}

As editors, we have had first-hand experience of the importance of diverse perspectives in illuminating values at a number of points in producing this book. As noted above, and as described in chapter 1, exposure to diversity of perspectives was important in the origins of the book. It was this that made us aware of the extent to which values-based practice itself had to that point been driven all unwittingly by values of individualism. We acknowledged our surprise at this illumination. The result, we said, is that the book owes its very existence to the diversity of perspectives on which it draws.

It is this same diversity operating now at the end of the book that was key to the outcomes we hope from the book. This is how this further illumination came about. As part of the process of co-writing we circulated drafts of the front and end matter for the book, including an earlier version of this concluding Chapter, to all authors for comment and input. Most, we were delighted to find, were well pleased. One contributor, however, Colin King (the lead author of chapter 46, "Beyond the Color Bar: Sharing Narratives in Order to Promote a Clearer Understanding of Mental Health Issues Across Cultural and Racial Boundaries"), made what at first came as a surprising observation. He pointed out that as editors we were 'speaking in the third person...' but commenting '... on first person accounts of the black and white cultural challenges...' with the result that '... your cultural values are absent.' 
In a subsequent conversation, Colin explained that he was drawing in this on Steven Gillard's observations (in chapter 46, "Beyond the Color Bar: Sharing Narratives in Order to Promote a Clearer Understanding of Mental Health Issues Across Cultural and Racial Boundaries") about personal pronoun use. Speaking in the third person about first personal experiences is a way of objectifying that experience while at the same time failing to 'see' our own values. In this case, Colin pointed out, we were at risk of failing to 'see' our own values as editors.

At one level, this might seem like old news. As we noted in the Forward to this book, it was our recognition that as editors (being all white, male and psychiatrists) we could not match the diversity of our contributors, that led to the book becoming a project in its own subject matter, values-based practice, operationalized through the co-writing procedures that in our writing workshops we went on to adopt. But this was precisely why Colin's observation was so important. For what his observation showed was that, notwithstanding the evident power of the values-based cowriting approach we had adopted, as evidenced by the quality of the contributions to the book, we as editors were still not able to 'see' our own values other than through the mirror of someone else's very different perspective. All of us are male. But as editors, we are white psychiatrists whereas Colin is a black writer and activist.

So there we have it. Generalizing Colin's observation takes us to the idea of an international open society of stakeholders in mental health as a key outcome from the book. As we describe below and in our Afterword, ${ }^{6}$ an international open society of stakeholders serves as a 'hall of mirrors' through which we can come to mutual understanding of the values driving mental health practice. True, we had explored that idea previously. But it had been an idea in theory only. Colin's observation of our inability to 'see' our own values made it real.

\subsubsection{Towards an 'Open Society' of Stakeholders in Mental Health}

Exposure to diversity of values may operate at different levels, personal, professional, national or, as here, international. Internationally, exposure to diversity of values requires the development of what the social psychiatrist, Jim Birley, drawing on his experience countering political abuses of psychiatry as President of the Geneva Initiative for Psychiatry, called an 'open society' among mental health stakeholders [6]. Now, some 40 years on, it is in taking us a step towards Jim Birley's open society, that the diversity of contributions to this book is provisionally

\footnotetext{
${ }^{6}$ Bill Fulford and Colin King have developed this idea in more detail with the philosopher Anna Bergqvist in their 'Hall of Mirrors: Towards an open society of mental health stakeholders in safeguarding against political abuse', (in English) in a special issue of the Polish journal of philosophy and psychiatry, Eidos, edited by Louis Sass. (Fulford, KWM., King, C., and Bergqvist, A., (2020) Eidos: a Journal for Philosophy of Culture. Volume 4, no 2, pps 23-38. https://doi.org/10.14394/ eidos.jpc.2020.0014)
} 
justified. Final justification will depend on Jim Birley's open society of mental health stakeholders becoming a reality. We return to how this is to be done in our Afterword.

\subsection{Conclusions}

In this chapter, we have described the values-based process we adopted in co-writing the book. This proved challenging emotionally and administratively. We addressed these challenges through two writing workshops and by way of many individual conversations and multiple exchanges of drafts. Although complex and difficult, we believe our co-writing approach to have been provisionally justified by the quality and range of narrative contributions this generated for the book. The diversity of perspectives represented by these contributions takes us an important step towards establishing the kind of open society among stakeholders that we have argued is required to support best practice in person-centred mental health care.

Which brings us, finally, to the 'mental health first' surprise we promised at the end of chapter 1 . The surprise, to anticipate, is that in establishing in this way an open society among stakeholders, mental health will be first in leading the way in person-centred care for health care as a whole.

This follows from the Science Driven Principle of values-based practice. As we saw in the introduction to Part IV (chapter 24, "Linking Science with People: An Introduction to Part IV, Science"), this Principle arises from the way in which advances in medical science and technology impact on practice. Such advances as we described open up new choices and with choices go values. David Crepaz-Keay and co-authors (chapter 28, "Journey into Genes: Cultural Values and the (Near) Future of Genetic Counselling in Mental Health") provide a vivid illustration of the impact of this principle in psychiatric genetics with their 'Journey into Genes'. But it is the full import of this principle for mental health that brings with it our 'mental health first' surprise. For if cultural values are a key factor in linking science with people in mental health today, then, tomorrow, with future developments in medical science and technology, cultural values will become a key factor also in linking science with people across health care as a whole.

That mental health should be first in recognizing and responding to this key connection between values and evidence comes as a surprise when it is set against the 'mental health second' stereotype of Twentieth Century health care. According to this stereotype, mental health ran always behind and in second place to other areas of health care. The 'mental health first' surprise, then, arising from the full impact of the Science Driven Principle of values-based practice, is that in Twenty-First Century health care, mental health, in being the first to recognize the need for an international open society of stakeholders, and engaging in this open society through personal narratives of the kind presented in this book, is leading the way for health care as a whole. Mental health is thus first in the field in developing the resources to 
support a model of shared clinical decision-making, based equally on evidence and on values, that is at the heart of best practice in contemporary person-centred clinical care.

\subsection{Guide to Further Information}

For sources on the various areas of philosophy relevant to a full justification of the importance of a diversity of value perspectives in mental health (noted at the start of Sect. 4 in this chapter), please see The Oxford Textbook of Philosophy and Psychiatry [7] - chapters of this textbook together with key readings are available for free download from the new website of the International Network for Philosophy and Psychiatry.

For a detailed reading guide and many training and other resources for valuesbased practice, please see the website for the Collaborating Centre for Values-based Practice in Health and Social Care at St Catherine's College, Oxford at: valuesbasedpractice.org.

As described further in our Afterword, the Collaborating Centre is kindly supporting the authors and editors of this book in taking forward the development of an international open society of stakeholders in mental health.

\section{References}

1. National Institute for Mental Health England. The National Framework of Values for Mental Health. Originally published on-line on the NIMHE website. Available on the values-based practice website (https://valuesbasedpractice.org) or in hard copy in Woodbridge K, Fulford KWM. 'Whose values?' A workbook for values-based practice in mental health care. London: The Sainsbury Centre for Mental Health (or as a free download https://atvaluesbasedparctice. org); 2004.

2. Diangelo R. White fragility: why its so hard for white people to talk about racism. Boston: Beacon Press; 2018.

3. van Voren R, Keukens R. Ch 48: Political abuse of psychiatry. In: Sadler JZ, van Staden W, Fulford KWM, editors. The Oxford handbook of psychiatric ethics. Oxford: Oxford University Press; 2015.

4. Fulford KWM, Dewey S, King M. Values-based involuntary seclusion and treatment: value pluralism and the UK's Mental Health Act 2007. Ch 60. In: Sadler JZ, van Staden W, Fulford KWM, editors. The Oxford handbook of psychiatric ethics. Oxford: Oxford University Press; 2015.

5. Fulford KWM, Smirnov AYU, Snow E. Concepts of disease and the abuse of psychiatry in the USSR. Br J Psychiatry. 1993;162:801-10.

6. Birley J. Psychiatric ethics: an International Open Society. Chapter 11. In: Dickenson D, Fulford KWM, editors. In two minds: a casebook of psychiatric ethics. Oxford: Oxford University Press; 2000. p. 327-35. 
7. Fulford KWM, Thornton T, Graham G. Progress in five parts. Chapter 1. In: Fulford KWM, Thornton T, Graham G, editors. The Oxford textbook of philosophy and psychiatry. Oxford: Oxford University Press; 2006. This book togeher with its associated readings will be available on open access and with free download on the new INPP Website that will be launched in early 2021.

Open Access This chapter is licensed under the terms of the Creative Commons Attribution 4.0 International License (http://creativecommons.org/licenses/by/4.0/), which permits use, sharing, adaptation, distribution and reproduction in any medium or format, as long as you give appropriate credit to the original author(s) and the source, provide a link to the Creative Commons license and indicate if changes were made.

The images or other third party material in this chapter are included in the chapter's Creative Commons license, unless indicated otherwise in a credit line to the material. If material is not included in the chapter's Creative Commons license and your intended use is not permitted by statutory regulation or exceeds the permitted use, you will need to obtain permission directly from the copyright holder.

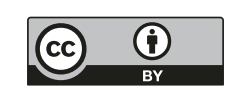

